\title{
Bias toward a 1:1 ratio in primer-introduced restriction analysis PCR: mechanism and minimization
}

S. Denden ${ }^{1}$, H. Abaidi' ${ }^{2}$ M.H. Hamdaoui ${ }^{2}$, J. Ben Chibani ${ }^{1}$ and

A. Haj Khelil ${ }^{1}$

${ }^{1}$ Research Unit on Biology and Molecular Anthropology Applied to Development and Health, Faculty of Pharmacy, University of Monastir, Monastir, Tunisia

${ }^{2}$ Research Unit on the Antioxidant Compounds, Oxidative Stress, Trace Elements and Metabolic Diseases,

École Supérieure des Sciences et Techniques de la Santé de Tunis, University of Tunis El-Manar, Tunis, Tunisia

Corresponding author: S. Denden

E-mail: denden_sabri@yahoo.fr

Genet. Mol. Res. 13 (3): 5686-5694 (2014)

Received March 5, 2014

Accepted June 30, 2014

Published July 25, 2014

DOI http://dx.doi.org/10.4238/2014.July.25.24

\begin{abstract}
Primer-introduced restriction analysis is widely used in molecular genetics. However, several studies have reported inconsistent data regarding sequencing, mainly among heterozygous samples. This discrepancy may be related to the bias towards a 1:1 ratio typically observed in heterozygous digestion products. In this study, we investigated the mechanism and minimization of this observed bias. Three mismatched polymerase chain reaction (PCR) models were analyzed by testing different PCR conditions and reaction mixtures. For EPHX1 gene rs1051740 single-nucleotide polymorphism PCR, DNA concentration, denaturation and elongation time, annealing temperature, and cycle number significantly influenced product ratios. For SERPINA1 gene PIMmalton deletion ( $\triangle \mathrm{Phe} 52)$ and $C H R N A 3$ gene
\end{abstract}


rs1051730 single-nucleotide polymorphism PCRs, significant bias fluctuations were observed only for the annealing temperature and cycle number conditions. The relevance of these results to the amplification efficiency parameter is discussed. Rather than reducing the observed bias, our data provide evidence of a counterbalance for preferential amplification, depending on cycle number, annealing temperature, and amplification efficiency alteration. Our results are relevant for application to primer-introduced restriction analysis PCR assays.

Key words: Amplification efficiency; Bias; Mismatched PCR; Primer-introduced restriction analysis PCR

\section{INTRODUCTION}

Primer-introduced restriction analysis (PIRA) polymerase chain reaction (PCR), also known as mismatched PCR, is a PCR method in which one or more mismatches are introduced into the primer sequence in order to create a restriction site for detecting a polymorphism/mutation. Although widely used in association studies and in the diagnosis of monogenetic diseases, many of the mismatched PCR genotyping assays reported show inconsistent data regarding sequencing, mainly among heterozygous genotypes (Hodanova et al., 1997; Keicho et al., 2001; Simsek et al., 2001). These discrepancies may be related to the bias toward the 1:1 ratio frequently observed in heterozygous digestion products, so that heterozygous samples may be mistaken as homozygous. The main cause at the origin of the observed bias is the amplification of different templates with mismatched primers. Indeed, it is well known that degenerate primers are associated with amplification bias, which is mainly related to the different primer binding energies in the non-perfectly matched priming sites of the mixed template (Kanagawa, 2003). However, in the case of mismatched PCR, different priming sites are not present in the templates, but the flanking region at the $5^{\prime}$ end of the mismatched primer contains different allelic versions [single-nucleotide polymorphisms (SNP) or others], which are likely responsible for the observed bias. Some previous studies have reported that primer hybridization efficiency may be influenced by sequence regions adjacent to the priming site (Zheng et al., 1996; Polz and Cavanaugh, 1998). Furthermore, primer-template secondary structure known as the dangling-end, which involves the first template nucleotide flanking the primer, has been shown to induce different primer-binding energies depending on the oligonucleotide sequence and the type of flanking nucleotide present (Bommarito et al., 2000). In PIRA PCR, the stability of secondary structures formed between a mismatched primer and a template may cause the observed bias. The duplex formed between a perfectly matched primer-template appears to be more stable. No amplification bias is observed in this case. However, other bias occurs in non-mismatched restriction fragment length polymorphism-PCR assay as the lower intensity of the digested band because of the smaller DNA content.

Different PIRA PCR assays described in previous studies did not show an apparent preference towards amplifying a particular type of polymorphism (A, T, C, and G), as inconsistent data were obtained. Furthermore, rather than involving the entire primer-template sequence in the pre-elongation step, different factors appear to be relevant in the over-repre- 
sentation of a particular sequence.

In this study, 3 mismatched PCR models were examined based on observed higher intensity of the non-digested band and compared to that of a digested band. Different PCR conditions and reaction mixtures were tested. Digestion products ratios were assessed. In this analysis, we examined the mechanism of observed PCR bias. We also investigated bias minimization in order to provide more accurate results by PIRA PCR.

\section{MATERIAL AND METHODS}

\section{Materials}

Three PIRA PCR assays for genotyping the EHPX1 gene rs1051740 SNP (Wong et al., 2000), the CHRNA3 gene rs1051730 SNP (this study), and the SERPINA1 gene PIMmalton deletion ( $\triangle \mathrm{TTC}$ ) (Denden et al., 2013) were chosen based on their generated band unconformities: non-digested bands were predominant among heterozygotes. Two non-mismatched PCR assays for the CYP1A2*1F gene rs762551 SNP (Cornelis et al., 2004) and the CHRNA3/5 locus rs8034191 SNP (this study) genotyping were used as controls. Three heterozygous samples for each locus were selected under classical genotyping conditions to assess the effect of PCR conditions on the observed bias.

\section{Genotyping}

In all assays, PCR components for the $25-\mu \mathrm{L}$ reaction mixtures included: $0.4 \mu \mathrm{M}$ of each primer, 50-200 ng genomic DNA, $200 \mu \mathrm{M}$ dNTP mix, $1.5 \mathrm{mM} \mathrm{MgCl}_{2}, 0.5 \mathrm{U}$ GoTaq flexi DNA polymerase (Promega; Madison, WI, USA), and 1X buffer. PCR cycling conditions were also similar, except for the hybridization temperature $\left(T_{H}\right)$ used. Amplification was carried out in a DNA Thermal Cycler (Multigene; Labnet; Edison, NJ, USA) according to the following program: $7 \mathrm{~min}$ at $94^{\circ} \mathrm{C} ; 35$ cycles of: $30 \mathrm{~s}$ at $94^{\circ} \mathrm{C}, 30 \mathrm{~s}$ at $\mathrm{T}_{\mathrm{H}}$, and $30 \mathrm{~s}$ at $72^{\circ} \mathrm{C}$; final extension for $7 \mathrm{~min}$ at $72^{\circ} \mathrm{C}$. The primers and $\mathrm{T}_{\mathrm{H}}$ used at each locus were (introduced mismatch is underlined): rs1051740 EHPX1 SNP: 5'-GATCGATAAGTTCCGTTTCACC$3^{\prime}$ and 5'-ATCTTAGTCTTGAAGTGAGGAT-3', $\mathrm{T}_{\mathrm{H}}=56^{\circ} \mathrm{C}$; rs 1051730 CHRNA3 SNP: 5'-GATGACGATGGACAAGGTTAC-3' and 5'-TCATCAAAGCCCCAGGTTA-3', $\mathrm{T}_{\mathrm{H}}=$ $56^{\circ} \mathrm{C}$; PIMmalton mutation SERPINA1: 5'-ACACCAGTCCAACAGCACCAATAAC-3' and 5'-TCTCCGTGAGGTTGAAATTCAGGCC- 3 ', $\mathrm{T}_{\mathrm{H}}=58^{\circ} \mathrm{C}$; rs762551 CYP1A2*1F gene SNP: 5'-CCCAGAAGTGGAAACTGAGA-3' and 5'-GGGTTGAGATGGAGACATTC-3'; $\mathrm{T}_{\mathrm{H}}=$ $60^{\circ} \mathrm{C}$; rs8034191 CHRNA3/5 locus SNP: 5'-GAGCAGGTTCATAATTCCAAGTG-3' and 5'-TGTAGTCCCAGCTACTCAGCAG $-3^{\prime} ; \mathrm{T}_{\mathrm{H}}=56^{\circ} \mathrm{C}$. Next, $10 \mu \mathrm{L}$ PCR products were digested overnight at $37^{\circ} \mathrm{C}$ with $2.5 \mathrm{U}$ of the corresponding restriction enzyme to each locus [EcoRV, AanI, MboII (Fermentas; Vilnius, Lithuania), ApaI, and Hpy188I (New England Biolabs; Ipswich, MA, USA), respectively] and electrophoresed on a 3\% agarose gel stained with ethidium bromide.

\section{Variables tested}

The PCR components and cycling conditions, previously assessed to show bias toward 1:1 ratio reduction, were evaluated. Each parameter was tested individually, while other 
conditions were maintained as in classical genotyping assays. The reaction mixture variables tested included: DNA amount [initial concentration (IC); IC x 2; IC / 2; IC / 4] and primer concentrations $(0.2,0.4$, and $0.8 \mu \mathrm{M})$. Different cycling parameters were analyzed, including denaturation time ( $30 \mathrm{~s}, 1 \mathrm{~min}, 1.5 \mathrm{~min})$, annealing time ( $30 \mathrm{~s}, 1 \mathrm{~min}, 1.5 \mathrm{~min})$, extension time (30 s, $1 \mathrm{~min}, 1.5 \mathrm{~min})$, cycle number $(25,27,30$, and 35$)$, and annealing temperature $\left(43^{\circ}-\right.$ $66^{\circ} \mathrm{C}$ gradient).

The investigation of mismatch and type effects on the observed bias was possible for the PIMmalton heterozygous genotype. Three primers sets were tested. The difference between the primers described above for PIMmalton genotyping was in terms of nucleotide type at the mismatched position (T, C, and G). PCR products were then analyzed on a 15\% (19:1) non-denaturant polyacrylamide gel to distinguish between amplified templates.

\section{Data analysis}

PCR and digestion products band intensities were quantified using the ImageJ software version 1.46r (available at http://imagej.nih.gov/ij/download.html). Digested/undigested products ratios were assessed through restriction fragment length polymorphism-PCR analysis. For PIMmalton PCR product analysis, deletion allele/normal allele ratios were used. Data are reported as means \pm standard deviation and compared using the Student $t$-test.

\section{RESULTS}

Digestion product ratio variations, according to variables tested, are shown in Figure 1. For the EPHX1 gene PCR, DNA concentration, denaturation and elongation times, annealing temperature, and cycle number significantly influenced the product ratios. Lower DNA concentrations only reduced the product ratios (IC x 2: ratio of $0.504 \pm 0.02$; IC / 4: ratio of $0.703 \pm 0.02 ; \mathrm{P}<0.001)$, whereas ratios were completely inverted for other variants: denaturation time ( $30 \mathrm{~s}$ : ratio of $0.595 \pm 0.04 ; 1.5 \mathrm{~min}$ : ratio of $1.113 \pm 0.19 ; \mathrm{P}<0.001)$, elongation time (30 s: ratio of $0.595 \pm 0.04 ; 1.5 \mathrm{~min}$ : ratio of $1.604 \pm 0.21 ; \mathrm{P}<0.001)$, annealing temperature $\left(60^{\circ} \mathrm{C}\right.$ : ratio of $0.176 \pm 0.01 ; 43^{\circ} \mathrm{C}$ : ratio of $\left.2.990 \pm 0.26 ; \mathrm{P}<0.001\right)$, and cycle number (35: ratio of $0.595 \pm 0.04 ; 25$ : ratio of $4.030 \pm 0.33 ; \mathrm{P}<0.001$ ). For the SERPINA1 gene PCR, significant bias fluctuations were only observed for annealing temperature and cycle number conditions. The product ratios were reduced at lower cycle numbers (35: ratio of $0.225 \pm 0.01$; 25: ratio of $0.858 \pm 0.04 ; \mathrm{P}<0.001)$ and inverted at low annealing temperatures $\left(65^{\circ} \mathrm{C}\right.$ : ratio of $0.106 \pm 0.01 ; 48^{\circ} \mathrm{C}$ : ratio of $\left.1.570 \pm 0.16 ; \mathrm{P}<0.001\right)$. For the $C H R N A 3$ gene $\mathrm{PCR}$, only bias reduction, occurring when lowering annealing temperature and cycles number, was observed (annealing temperature $57^{\circ} \mathrm{C}$ : ratio of $0.077 \pm 0.006 ; 47^{\circ} \mathrm{C}$ : ratio of $0.419 \pm 0.01 ; \mathrm{P}<0.001$ ), cycle number (35: ratio of $0.083 \pm 0.007 ; 27$ : ratio of $0.411 \pm 0.01 ; \mathrm{P}<0.001$ ). No significant variation in product ratios was observed for any of the non-mismatched PCR assays.

Mismatch and type effects in bias extent analysis using PIMmalton heterozygous PCR is shown in Figure 2. The results revealed a significant deviation towards a 1:1 ratio among mismatched PCRs (ratio mean of $0.228 \pm 0.01$ ) compared with non-mismatched assays (ratio of $1.02 \pm 0.03, \mathrm{P}<0.001$ ). However, there was no significant difference between the mismatches tested (A:A, ratio of $0.225 \pm 0.01 ; \mathrm{A}: \mathrm{G}$, ratio of $0.216 \pm 0.01 ; \mathrm{A}: \mathrm{C}$, ratio of 0.243 $\pm 0.02 ; \mathrm{P}>0.05)$. 

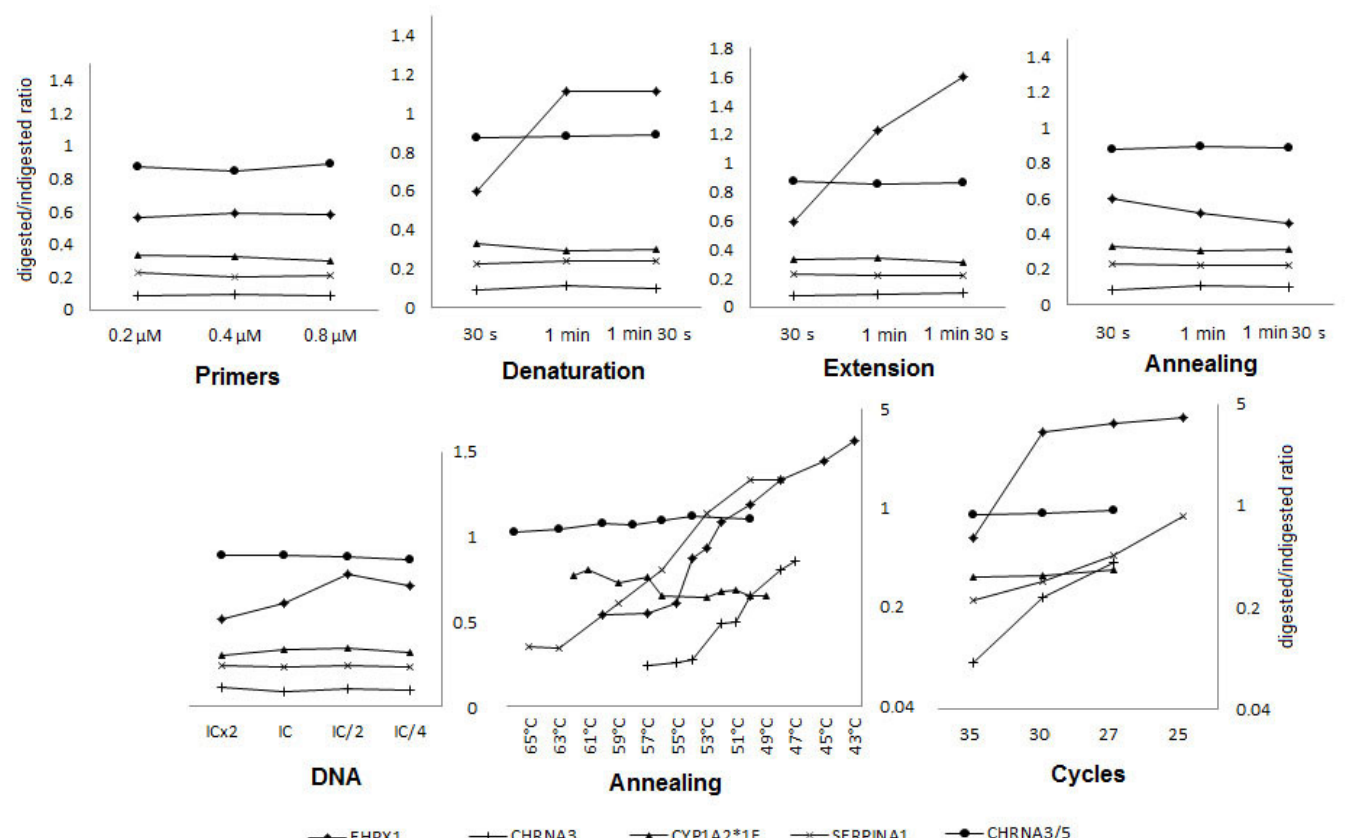

Figure 1. Digestion product ratio variations according to primers and DNA concentrations, denaturation, annealing and extension times, annealing temperature, and cycle number. IC = initial concentration.

non-

Mismatched PCRs

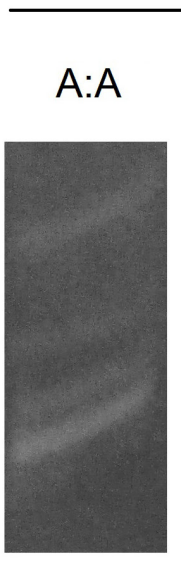

$A: G$

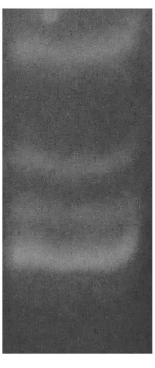

\section{Mismatched}

$A: C$

$A: T$
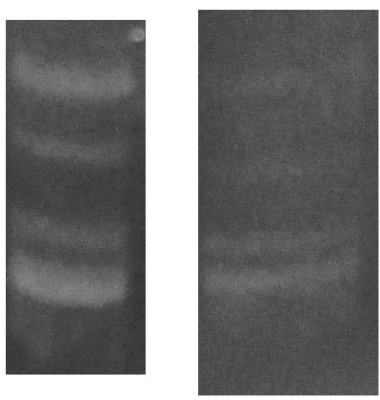

Figure 2. Mismatch and type effects on bias extent analysis using the PIMmalton heterozygous PCR. The amplification of normal allele (non-deleted) showed a 134-bp band whereas the PIMmalton allele (3-bp deletion) was shown as a 131-bp band. Heteroduplex bands are artifacts of small-deletion PCR amplification. 


\section{DISCUSSION}

Amplification bias observed using PIRA PCR may be related to primer-template thermodynamic fluctuations. No apparent preference in the amplification of a type of polymorphism was observed. Indeed, differences in primer-binding energies, which is at the origin of the observed bias, are target- and flanking region sequence context-dependent (Zheng et al., 1996; Polz and Cavanaugh, 1998; Bommarito et al., 2000). In the present study, the assignment of primer mismatch as an essential condition in bias occurrence was confirmed; the non-mismatched PCR assay showed no deviation towards a 1:1 ratio (ratio of $1.02 \pm 0.03$ ), whereas bias was observed in all mismatches tested (ratios of $0.228 \pm 0.01$ ). However, the mismatch type showed no significant effect on bias extent (A:A, ratio of $0.225 \pm 0.01$; A:G, ratio of $0.216 \pm 0.01 ; \mathrm{A}: \mathrm{C}$, ratio of $0.243 \pm 0.02 ; \mathrm{P}>0.05$ ). Several reports demonstrated that different internal mismatch types may influence, to different extents, duplex thermodynamics (Allawi and SantaLucia, 1997, 1998; Peyret et al., 1999). However, they also reported that the contribution to duplex stability is context-dependent. In contrast, any mismatch type inducing thermodynamic fluctuation may not have a significant influence on the observed bias.

According to previous studies, amplification bias depends on a number of variables, both intrinsic and extrinsic to the PCR. One of the most important is amplification efficiency, a parameter that depends on template quality, concentration, primer length, binding energy, replicate length, and PCR reagents availability, among others (Suzuki and Giovannoni, 1996; Polz and Cavanaugh, 1998; SantaLucia, 2007; Gonzalez et al., 2012). The alteration of this parameter may lead to controversial results. In our study, this condition was tested at lower concentrations $(0.08 \mu \mathrm{M})$ of one primer. In addition to obtaining a weaker yield, the overamplified alleles were reversed in the cases of EHPX1 (ratio of 2.306 \pm 0.22 ) and SERPINA1 (ratio of $1.438 \pm 0.11$ ) PCRs. No yield was obtained for the CHRNA3 PCR under these conditions. Amplification efficiency may also be altered, to different extents, by lower DNA concentrations or expanding denaturation and elongation times, explaining the deviation in the product ratios observed with EHPXI PCR. Polz and Cavanaugh (1998) reported bias reduction using high template concentrations. This finding does not contradict our results, as low DNA concentration-induced amplification efficiency alterations can cause stronger bias compared to higher DNA amounts. In another study, Kurata et al. (2004) observed unexplained PCR bias when using a longer extension time (180 s), which may be attributable to amplification efficiency alteration.

Under optimal PCR conditions, PCR selection may favor one of the alleles from the beginning of the amplification. However, it has been demonstrated that the bias mechanism can vary during amplification and according to amplification efficiency. Suzuki and Giovannoni (1996) suggested a kinetic model to explain the observed bias. They reported that in multitemplate reactions with higher amplification efficiency, the favored template will reach saturation concentration (a plateau phase resulting from the inhibitory template reannealing effect) earlier than the other templates, which are still increasing. Based on this observation, the final over-amplified product may correspond to the actual favored allele or to which amplification is still occurring, depending on the amplification cycle number. This may explain the results obtained for EPHXI PCR. Our experiments showed that the over-amplified allele at 35 cycles (non-digested allele) was not observed for less than 27 PCR cycles. However, this finding was not observed for CHRNA3 and SERPINA1 PCR, and bias reduction was observed 
when the number of PCR cycles was lowered. Suzuki and Giovannoni (1996) also reported that PCR with lower amplification efficiency does not show an inhibitory template reannealing effect. This observation agrees with our data. The CHRNA3 and SERPINA1 PCRs showed lower yield compared to that of the EPHXI gene, performed under the same reaction and cycling conditions. However, one limit of the technique used in this study for bias quantification (ethidium bromide staining) should be considered. This technique does not allow for bias quantification of PCR involving fewer than 25 cycles, which is needed for further investigation of the CHRNA3 and SERPINA1 PCRs. Several studies investigating bias in PCR amplification of mixed templates also showed that a reduction in the number of PCR cycles may reduce the observed bias (Suzuki and Giovannoni, 1996; Polz and Cavanaugh, 1998; Sipos et al., 2007). However, this study is the first to report a preferential amplification counterbalance, depending on cycle number.

The annealing temperature relationship with PCR bias was previously investigated (Ishii and Fukui, 2001; Sipos et al., 2007). The data reported examined mismatched primingsite-induced bias, and observed amplification of bacterial strain template mixtures. In their study, Ishii and Fukui (2001) reported reduced bias with lower annealing temperature only in the case of the mismatched templates (P1100 and M1100) whereas they did not observe any change with perfectly matched templates (P907 and M907) even presenting an amplification bias. The result obtained indicates that bias toward a 1:1 ratio may also occur with non-mismatched primers, like when sequences flanking the priming site have a strong influence on primer-binding energies. Furthermore, a deeper analysis of the results presented by Ishii and Fukui (2001) revealed that, in the case of the P1100 and M1100 templates, overamplification of one template at higher annealing temperature $\left(55^{\circ} \mathrm{C}\right.$ : ratio of $\left.0.17 \pm 0.08\right)$ was counterbalanced at lower annealing temperature $\left(45^{\circ} \mathrm{C}\right.$ : ratio of $\left.1.47 \pm 0.21\right)$. A bias reduction also occurred for P907 and M907 templates (the product ratio was reduced from $2.12 \pm 0.12$ at $55^{\circ} \mathrm{C}$ to $1.61 \pm 0.13$ at $45^{\circ} \mathrm{C}$ ). Zheng et al. (1996), who investigated universal oligonucleotide hybridization using small subunit rRNA, reported that bias between templates resulted from fluctuations in the dissociation temperature, a parameter defined as the temperature at which $50 \%$ of the duplex remains intact during the hybridization washing period. In addition to these observations showing reduced bias by lowering the hybridization temperature, we further observed for the S-*-Univ-1390-a-A-18 probe that the preference in template hybridization at high temperature $\left(55^{\circ} \mathrm{C}\right)$ was counterbalanced at lower temperature $\left(44^{\circ} \mathrm{C}\right)$. For the S-*-Univ-1392-a-A-15 probe, bias reduction was only observed when the hybridization temperature decreased from $47^{\circ} \mathrm{C}$ to $40^{\circ} \mathrm{C}$. These findings are similar to those observed in our study. Over-amplification of the non-deleted allele of the SERPINA1 gene and the nondigested allele of the $E P H X 1$ gene at $65^{\circ} \mathrm{C}$ (ratio of $0.106 \pm 0.01$ ) and $60^{\circ} \mathrm{C}$ (ratio of $0.176 \pm$ 0.01 ) were counterbalanced at $48^{\circ} \mathrm{C}$ (ratio of $1.570 \pm 0.16$ ) and $43^{\circ} \mathrm{C}$ (ratio of $2.990 \pm 0.26$ ), respectively. In addition, bias reduction was only observed for the CHRNA3 gene PCR when the annealing temperature was decreased from $57^{\circ} \mathrm{C}$ (ratio of $0.077 \pm 0.006$ ) to $47^{\circ} \mathrm{C}$ (ratio of $0.419 \pm 0.01$ ). Testing of a lower annealing temperature was not possible in this case as no yield was obtained.

According to these data, for PCR cycle number, the amplification preference may also be dependent on annealing temperature. Two mechanisms may be involved in the bias reduction and/or template preference counterbalance by lowering the annealing temperature. First, the variance between templates in term of primer-binding energy may be largely affected 
by the low annealing temperature, allowing for more extensive hybridization independently from the template preference. Second, low temperature promotes non-specific hybridization in other loci. This condition may alter the amplification efficiency of the specific replicate, which can induce a preference counterbalance, as observed in our experiments.

Various factors should be considered in PIRA PCR assays. Internal factors, specific to the primers and templates, may be easily controlled throughout PCR cycling and annealing temperature parameter optimization. However, external variables such as DNA concentration, quality, DNA salt concentration, and PCR reagent concentrations appear to alter to varying extents, depending on the optimization of internal factors. Fluctuations between samples may not be completely avoided, as they depend on the amplification efficiency in each tube. Welldefined experimental conditions are needed using DNA samples extracted under the same conditions and having the same $\mathrm{pH}$ and salt concentrations. Indeed, these last variables are well known to impact DNA thermodynamics (Allawi and SantaLucia, 1998; SantaLucia, 1998). PCR drift may also occur from pipetting errors, which can be avoided by a master-mix and through increased experimenter vigilance.

In conclusion, our study revealed that both PCR selection and drift contribute to the bias observed in mismatched PCR assays, which require PCR cycling and annealing temperature optimization and stringent experimental conditions in order to be reduced.

\section{REFERENCES}

Allawi HT and SantaLucia J Jr (1997). Thermodynamics and NMR of internal G.T mismatches in DNA. Biochemistry 36: 10581-10594.

Allawi HT and SantaLucia J Jr (1998). Nearest-neighbor thermodynamics of internal A.C mismatches in DNA: sequence dependence and $\mathrm{pH}$ effects. Biochemistry 37: 9435-9444.

Bommarito S, Peyret N and SantaLucia J Jr (2000). Thermodynamic parameters for DNA sequences with dangling ends. Nucleic Acids Res. 28: 1929-1934.

Cornelis MC, El-Sohemy A and Campos H (2004). Genetic polymorphism of CYP1A2 increases the risk of myocardial infarction. J. Med. Genet. 41: 758-762.

Denden S, Lakhdar R, Keskes NB, Hamdaoui MH, et al. (2013). PCR-based screening for the most prevalent alpha 1 antitrypsin deficiency mutations (PI S, Z, and Mmalton) in COPD patients from Eastern Tunisia. Biochem. Genet. 51: 677-685.

Gonzalez JM, Portillo MC, Belda-Ferre P and Mira A (2012). Amplification by PCR artificially reduces the proportion of the rare biosphere in microbial communities. PLoS One 7: e29973.

Hodanova K, Hrebicek M, Cervenková M, Aerts JM, et al. (1997). Incorrect assignment of N370S mutation status by mismatched PCR/RFLP method in two Gaucher patients. J. Inherit. Metab. Dis. 20: 611-612.

Ishii K and Fukui M (2001). Optimization of annealing temperature to reduce bias caused by a primer mismatch in multitemplate PCR. Appl. Environ. Microbiol. 67: 3753-3755.

Kanagawa T (2003). Bias and artifacts in multitemplate polymerase chain reactions (PCR). J. Biosci. Bioeng. 96: 317-323.

Keicho N, Emi M, Kajita M, Matsushita I, et al. (2001). Overestimated frequency of a possible emphysema-susceptibility allele when microsomal epoxide hydrolase is genotyped by the conventional polymerase chain reaction-based method. J. Hum. Genet. 46: 96-98.

Kurata S, Kanagawa T, Magariyama Y, Takatsu K, et al. (2004). Reevaluation and reduction of a PCR bias caused by reannealing of templates. Appl. Environ. Microbiol. 70: 7545-7549.

Peyret N, Seneviratne PA, Allawi HT and SantaLucia J Jr (1999). Nearest-neighbor thermodynamics and NMR of DNA sequences with internal A.A, C.C, G.G, and T.T mismatches. Biochemistry 38: 3468-3477.

Polz MF and Cavanaugh CM (1998). Bias in template-to-product ratios in multitemplate PCR. Appl. Environ. Microbiol. 64: 3724-3730.

SantaLucia J Jr (1998). A unified view of polymer, dumbbell, and oligonucleotide DNA nearest-neighbor thermodynamics. Proc. Natl. Acad. Sci. U. S. A. 95: 1460-1465.

SantaLucia J Jr (2007). Physical principles and visual-OMP software for optimal PCR design. Methods Mol. Biol. 402: 3-34. 
Simsek M, Tanira MO, Al-Baloushi KA, Al-Barwani HS, et al. (2001). A precaution in the detection of heterozygotes by sequencing: comparison of automated DNA sequencing and PCR-restriction fragment length polymorphism methods. Clin. Chem. 47: 134-137.

Sipos R, Székely AJ, Palatinszky M, Révész S, et al. (2007). Effect of primer mismatch, annealing temperature and PCR cycle number on 16S rRNA gene-targetting bacterial community analysis. FEMS Microbiol. Ecol. 60: 341-350.

Suzuki MT and Giovannoni SJ (1996). Bias caused by template annealing in the amplification of mixtures of 16S rRNA genes by PCR. Appl. Environ. Microbiol. 62: 625-630.

Wong NA, Rae F, Bathgate A, Smith CA, et al. (2000). Polymorphisms of the gene for microsomal epoxide hydrolase and susceptibility to alcoholic liver disease and hepatocellular carcinoma in a Caucasian population. Toxicol. Lett. 115: $17-22$.

Zheng D, Alm EW, Stahl DA and Raskin L (1996). Characterization of universal small-subunit rRNA hybridization probes for quantitative molecular microbial ecology studies. Appl. Environ. Microbiol. 62: 4504-4513. 